\title{
Microbiological profile and antibiotic resistance pattern of urinary tract infection in a tertiary care hospital
}

Sir,

Urinary tract infection (UTI), of varying severity is one of the most commonly encountered ailments in nephrology outpatient department (OPD). The commonest pathogens causing community acquired UTI (CAUTI) are E. Coli, Proteus mirabilis, Pseudomonas aeruginosa and Klebsiella pneumonia. ${ }^{1}$ There is a rapidly rising trend of antibiotic resistance among these uropathogens, may be due to indiscriminate antibiotic usage and poor patient compliance. We conducted a retrospective analysis of all culture proven UTI cases that attended the nephrology
OPD of S. K. hospital, Trivandrum from August 2017 to July 2019 (24 months). 130 males and 121 females were included in the study. The mean age of the study group was 62.8 years with a male predominance in the age group above 50 years and a female predisposition in less than 50 years group. Commonest pathogen isolated in urine culture was E. coli $(52.98 \%)$ followed by Klebsiella (39.09\%), Citrobacter (8.76\%), Pseudomonas (8.37\%), Enterobacter (2.39\%), Enterococci (1.99\%), Candida (1.59\%), Acinetobacter (1.2\%), Proteus (1.2\%), Streptococci $(1.2 \%)$ and Serratia $(0.4 \%)$.

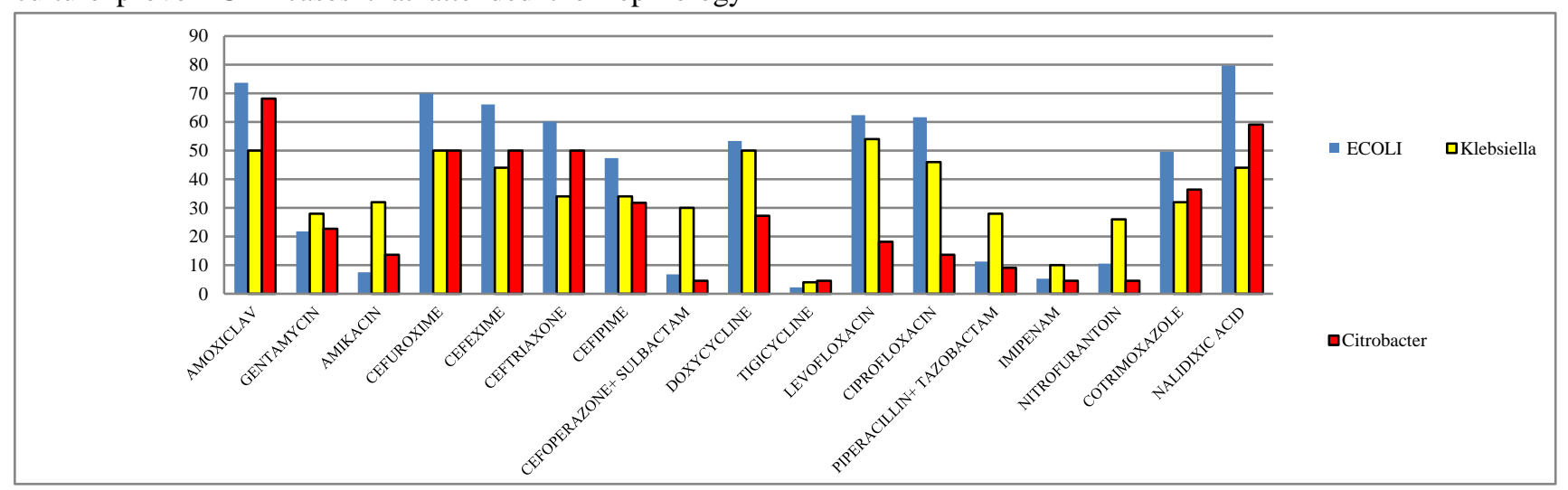

Figure 1: Antibiotic resistance pattern of E, coli, Klebsiella and Citrobacter.

We noticed the emergence of Citrobacter as a prominent uropathogen in CAUTI cases, especially in those with diabetes mellitus. Citrobacter was previously described primarily as a nosocomial pathogen in elderly hospitalized patients who underwent some form of endurological procedures or having obstructive uropathy. ${ }^{2}$ The study revealed a significant resistance to amino-penicillin, cephalosporins, quinolones and cotrimoxazole by common uropathogens. Least resistance was noted for aminoglycosides, Imipenem and nitrofurantoin. Suggest 7day course of nitrofurantoin for empirical treatment of CAUTI. Our study contributes to the expanding literature on antibiotic resistance pattern of uropathogens and aids in generating evidence-based recommendation for empirical management of CAUTI.

\section{Vismaya V. R. ${ }^{1 *}$, Naiema Shajihan ${ }^{1}$, Midhun Ramesh ${ }^{2}$, Ambika Devi $^{3}$}

${ }^{1}$ Department of Pharmacy practice, The Dale View College of Pharmacy and Research Centre, Trivandrum, Kerala, India
${ }^{2}$ Genesis Institute of Medical Sciences, Kannur, Kerala, India

${ }^{3}$ Department of Microbiology, S. K. Hospital, Trivandrum, Kerala, India

*Correspondence to

Dr. Vismaya V. R., E-mail: vismayavijayaram@gmail.com

\section{REFERENCES}

1. Kumar MS, Ghosh S, Nayak S, Das AP. Recent advances in biosen-sor based diagnosis of urinary tract infection. Biosens Bioelectron. 2016;80:497510.

2. Ranjan KP, Ranjan, N. Citrobacter: An emerging health care associated urinary pathogen. Urol ann. 2013;5(4):313-14.

Cite this article as: Vismaya VR, Shajihan N, Ramesh M, Devi A. Microbiological profile and antibiotic resistance pattern of urinary tract infection in a tertiary care hospital. Int J Basic Clin Pharmacol 2021;10:611-1. 